\title{
Patterns of cerebral activation during lexical and phonological reading in Portuguese
}

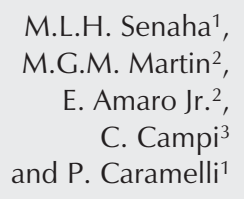

\author{
${ }^{1}$ Grupo de Neurologia Cognitiva e do Comportamento, \\ Departamento de Neurologia, ${ }^{2}$ Instituto de Radiologia, \\ ${ }^{3}$ Instituto do Coração, Faculdade de Medicina, \\ Universidade de São Paulo, São Paulo, SP, Brasil
}

\section{Correspondence \\ M.L.H. Senaha \\ Rua Montesquieu, 371/62 \\ Chácara Klabin \\ 04116-190 São Paulo, SP \\ Brasil \\ Fax: +55-11-3251-3698 \\ E-mail: senaha@uol.com.br \\ M.L.H. Senaha held a scholarship from FAPESP (No. 99-1067-2) \\ from 1999 to 2001. M.G.M. Martin received financial support from CAPES (No. BEX1341/04-9) from 2004 to 2005.}

Received March 3, 2004

Accepted August 5, 2005

\begin{abstract}
According to the concepts of cognitive neuropsychology, there are two principal routes of reading processing: a lexical route, in which global reading of words occurs and a phonological route, responsible for the conversion of the graphemes into their respective phonemes. In the present study, functional magnetic resonance imaging (fMRI) was used to investigate the patterns of cerebral activation in lexical and phonological reading by 13 healthy women with a formal educational level greater than 11 years. Participants were submitted to a silent reading task containing three types of stimuli: real words (irregular and foreign words), nonwords and illegitimate graphic stimuli. An increased number of activated voxels were identified by fMRI in the word reading (lexical processing) than in the nonword reading (phonological processing) task. In word reading, activation was greater than for nonwords in the following areas: superior, middle and inferior frontal gyri, and bilateral superior temporal gyrus, right cerebellum and the left precentral gyrus, as indicated by fMRI. In the reading of nonwords, the activation was predominant in the right cerebellum and in the left superior temporal gyrus. The results of the present study suggest the existence of differences in the patterns of cerebral activation during lexical and phonological reading, with greater involvement of the right hemisphere in reading words than nonwords.
\end{abstract}

Key words

- Functional magnetic resonance imaging

- Reading

- Lexical processing

- Phonological processing

- Cerebral activation

\section{Introduction}

Systematic studies of reading disturbances began at the end of the 19th century with the investigations conducted by the neurologists Wernicke, Charcot and Déjerine. This period was marked by the search for the neuroanatomic substrates responsible for the mental functions and the characterization of syndromes through the description and associa- tion of symptoms (1,2). Since 1970, with the concept of cognitive neuropsychology, numerous studies have attempted to understand the processes involved in several cognitive abilities (3-10). More recently, studies involving resources of functional neuroimaging have started to reintegrate the knowledge related to the cognitive functions with the neural substrate. The exchange of knowledge between cognitive neuropsychology and 
functional neuroimaging has been illustrated in several studies (11-16).

Cognitive neuropsychology aims to understand the function of a normal or damaged brain through models of information processing. Cognitive models of reading postulate the existence of two main routes of processing, a lexical one and a phonological one. In the phonological route, also called perilexical route, the segmentation from the graphic stimuli (e.g., word) to sublexical components (e.g., letters) occurs and each letter/grapheme is converted to its respective sound/phoneme. This type of reading may occur for low-frequency and low-familiar regular words. This kind of reading process is essential for nonword reading, for instance, "ced". In the lexical route, the word is read without the decomposition from the written stimuli to sublexical components, the word is read as a whole and the access to the semantic system may happen. Only previously known words can be read by the lexical route. Frequent and familiar words tend to be processed by the lexical route and the appropriate pronunciation of irregular words like "pint" depends on the lexical reading. Therefore, the linguistic variables of the stimuli determine or facilitate the type of reading processing to be done. Irregular words must be read by the lexical route, and nonwords by the phonological route.

The central, linguistic disturbances of reading can be divided into two groups: the first includes patients who predominantly read using the phonological route through the rules of grapheme-phoneme conversion, and the second includes those who read the words globally, that is, using the lexical route. Surface dyslexia belongs to the first group and the deep, phonological and semantic dyslexias belong to the second (1719).

Phonological dyslexia is characterized by the relative preservation of the lexical route with respect to a disturbance of the phonological reading responsible for the grapheme-phoneme conversion. For this reason, the individuals with phonological dyslexia can read frequent irregular words lexically whereas they are not capable of reading nonwords, unknown or low-frequency words. In contrast, surface dyslexia preserves reading ability by the phonological route, while the lexical route is impaired. The individuals with surface dyslexia read nonwords and regular words through the graphemephoneme conversion route, but they have difficulties in reading irregular words. These individuals, due to damage to the lexical mechanisms involved in reading, read the irregular words through the phonological route, applying the grapheme-phoneme conversion rules, and consequently presenting

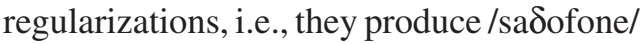
when trying to read the written word <saxophone $>$. The verification of these two different types of dyslexia is considered to be an example of double dissociation. Thus, from the observation of patients with surface and phonological dyslexias, it is concluded that the phonological and lexical routes involve different cognitive mechanisms and it is assumed that the neural mechanisms responsible for these processes are at least partially different.

One of the first studies involving reading and functional neuroimaging was conducted by Petersen et al. (11). The authors used a positron emission tomography (PET) technique to determine orthographic effects in eight right-handed individuals. They used four types of stimuli: real words (single common nouns), pseudowords (letter strings that obey the rules of English orthography), unpronounceable consonant letter strings, and false letters and they identified the activation of the lateral extrastriate cortex in the four groups of stimuli. Words and pseudowords produced activation of the medial extrastriate cortex on the left. Comparing the activation in the passive presentation of real words and pseudowords, the activation of the left frontal cortex was verified during the 
silent reading of real words, suggesting that the prefrontal area on the left could be involved in the semantic processing of words.

Rumsey et al. (14), in another PET study, examined the orthographic (lexical) and phonological processing of 14 right-handed men. Even in reading aloud tasks as well as in lexical decision, activation was lateralized to the left hemisphere, including the following areas: lingual and fusiform gyri, periRolandic cortex, thalamus, and anterior cingulate. Reading aloud real words and nonwords activated the left superior temporal gyrus with a more significant activation during the phonological than the lexical (orthographic) reading. The authors concluded that their results supported the connectionist models of reading since they did not reveal differences in the activation during the reading of real words and nonwords, and suggested that the processing of real words as well as of nonwords occurs inside a common neural network.

Different results were obtained in another study (20) using functional magnetic resonance imaging (fMRI), in which 38 individuals were submitted to four different tasks involving graphic stimuli. One of the tasks involved phonological processing (nonword rhyme judgment) and another involved lexical-semantic processing (semantic category judgment). The researchers observed activation during phonological processing in the inferior frontal gyrus and temporal lobe (with unilateral activation of the inferior frontal gyrus in men, but bilateral activation in women). During the lexical-semantic processing, the important areas were the middle and superior temporal gyri of both hemispheres.

Considering the Cognitive Neuropsychology approach regarding the different reading processes in patients with brain lesions the existence of double dissociation (phonological dyslexia versus surface dyslexia) suggests that the phonological and lexical routes involve different cognitive mechanisms and, probably, different neural substrates are responsible for these processes. The objective of the present study was to investigate the patterns of cerebral activation by fMRI during lexical and phonological reading in 13 healthy females. We hypothesized that the cerebral activation patterns differ according to the different types of reading process.

\section{Subjects and Methods}

\section{Subjects}

Thirteen healthy right-handed women aged 18 -36 years (mean $=25.2$ years) participated in the study. All individuals were readers of the Portuguese language, with a minimum of 12 years of education and with the maximum possible mark in the Edinburgh Handedness Questionnaire (21). No history of neurological disease or of learning difficulties was reported and the subjects were not using any drug with action on the central nervous system. Subjects were selected through the application of a questionnaire that contained questions to determine if they fulfilled the inclusion criteria mentioned above. A brief reading task was applied for the exclusion of non-fluent readers. The study was approved by the Ethics Committee of the University Hospital and the individuals selected gave written informed consent to participate.

\section{Tasks}

Individuals performed a silent reading task during the acquisition of the images by fMRI. This silent reading task presented an $\mathrm{ABC}$ design whose objective was to identify the activation of lexical and phonological reading in the alphabetic system. The $\mathrm{ABC}$ design corresponds to a task that is composed of three different lists. Condition A corresponded to list 1 (real words - irregular and foreign words), condition B to list 2 (nonwords) and condition $\mathrm{C}$ to list 3 (non- 
readable graphic stimuli; see Table 1 for an example).

The first list (list 1) was composed of 50 real words, half of them consisting of irregular words in Portuguese and the other half consisting of foreign words. The words ranged from four to nine letters in length and all were concrete nouns. The stimuli of this list containing irregular words and foreign words, should be read by lexical processing.

The second list (list 2) consisted of 50 nonwords written in the alphabetic system and was elaborated from the stimuli of the first list. The consonants, but not the vowels, of each real word were modified, with nonwords with the same structure and syllabic extension thus being obtained. For instance: starting from the irregular word axila, the nonword abida was generated. The reading of nonwords should be accomplished by phonological processing, that is, by grapheme-phoneme conversion processing.

The third list (list 3) had 50 illegitimate graphic stimuli. These stimuli were not readable because they were formed by pseudoletters obtained through distortions of the letters. The illegitimate graphic stimuli were elaborated from the second list, with the lines of the letters being modified and served as controls for the stimuli of lists 1 and 2 . Therefore, in the analysis of the images from fMRI, these illegitimate graphic stimuli were used as a baseline condition, containing elements of visual and pre-linguistic processing.

As described above, the silent reading task resulted from the combination of three different lists, each containing 50 stimuli, involving real words, nonwords and illegitimate graphic stimuli. The 50 stimuli of each

Table 1. Stimuli used in the silent reading task.

\begin{tabular}{cccc}
\hline List & Type of stimulus & Reading processing & Examples \\
\hline 1 & Words (irregular and foreign words) & Lexical & AXILA \\
2 & Nonword & Phonological & ABIDA \\
3 & Illegitimate graphic stimuli & Without reading & EIIII! \\
\hline
\end{tabular}

list were distributed into five blocks of 10 stimuli each. Therefore, the task was divided into 15 blocks that were randomly presented. To minimize possible effects from habituation to the type of stimulus, each epoch contained $80 \%$ of the stimuli belonging to one condition and $10 \%$ of the stimuli belonging to each of the remaining conditions.

\section{Preparation of the stimuli for the reading task}

The graphic stimuli written in the alphabetic system were typed using the Microsoft Word program (Microsoft, Seattle, WA, USA), in capital letters of the Arial font type, size 90. All stimuli were transported from the Word to the Paint program where they appeared against a black background. The illegitimate graphic stimuli generated from the nonword letter distortions were elaborated in the Paint program using the "eraser" feature to fade part of the letters. Stimuli were presented as bitmap files to be used by the Visual Basic program version 4.0 (Microsoft).

The duration of stimulus presentation and the interval between stimuli were $1000 \mathrm{~ms}$. Stimulus presentation and image acquisition were synchronized via a TTL pulse (Zurc \& Zurc DataSystems, São Paulo, SP, Brazil) and the stimuli were projected by a multimedia projector. The subjects visualized the stimuli through a mirror (each letter size occupied 4" degrees in the visual field) attached to the head coil, and were instructed to read them silently.

After the silent reading task during the acquisition of the images, in order to verify if the subjects had adhered to the experiment, they were asked if they remembered the stimuli they had read. To confirm the capacity of lexical and phonological reading, a post-test of word and nonword reading aloud was applied. All individuals had adhered to the experiment and were perfectly capable of reading through lexical and phonological processes. 


\section{Image acquisition and data processing}

The images were obtained in a Horizon 1.5 Tesla magnet of the General Electric Medical Systems (Milwaukee, MN, USA), equipped with a $40 \mathrm{mT} / \mathrm{m}$ gradient, using the blood oxygen level-dependent (BOLD) contrast technique with echo planar imaging. Fifteen slices were collected. The images were oriented on the anterior commissureposterior commissure line, with 7-mm thick, 0.7-mm gap slices covering the whole brain. The time of repetition was $2000 \mathrm{~ms}$, the time to echo was $40 \mathrm{~ms}$, the field of view was 400 x $200 \mathrm{~mm}$, with a $90^{\circ}$ flip angle and a matrix of 128 x 64 voxels. A total of 2250 images were collected for each subject. Analysis was performed with a personal computer running Linux.

The data were first realigned (22) to minimize motion-related artifacts and smoothed with a Gaussian filter (full-width half-maximum - $7.2 \mathrm{~mm}$ ). Responses to the experimental paradigms were then detected by time-series analysis using gamma variate functions (peak responses at 4 and 8 s) to model the BOLD response. The analysis was set up as follows. First, each experimental condition was convolved separately with the 4- and 8-s Poisson functions to yield two models of the expected hemodynamic response to that condition. The weighted sum of these two convolutions that gave the best fit to the time series at each voxel was then computed. This weighted sum effectively allows voxel-wise variability in time to peak hemodynamic response. Following this fitting operation, a ratio of the sum of squares of deviations from the mean intensity value due to the model (fitted time series) divided by the sum of squares due to the residuals (original time series minus model time series) was calculated. This statistic is called the SSQratio. The percent change of the BOLD signal at each voxel was also calculated. This was done by ((fitmax - fitmin)/ mean signal intensity) x 100, where fitmax and fitmin were the maximum and minimum values of the fitted response for the time series in question. In order to sample the distribution of the SSQratio under the null hypothesis that observed SSQratio values were not determined by experimental design (with minimal assumptions), the time series at each voxel was permuted using a waveletbased resampling method described in detail by Bullmore et al. (23). This process was repeated 10 times at each voxel and the data were combined over all voxels, resulting in 10 permuted parametric maps of the SSQratio on each plane for each participant. The same permutation strategy was applied to each voxel to preserve spatial correlational structure in the data during randomization. Combining the randomized data over all voxels yields the distribution of the SSQratio under the null hypothesis. A test that any given voxel is activated at any required type I error can then be carried out by obtaining the appropriate critical value of the SSQratio from the null distribution. For example, SSQratio values in the observed data lying above the 99th percentile of the null distribution have a probability of $\leq 0.01$ under the null hypothesis. We have shown that this permutation method gives very good type I error control with minimal distributional assumptions $(23,24)$.

After these processes, two activation maps were created for each individual, one of them comparing real words to illegitimate graphic stimuli (A x C), and the other comparing nonwords to illegitimate graphic stimuli (B x C). The objective of this comparison was to study the word and nonword silent reading by eliminating (subtracting) the activations of the neural structures responsible for the primary and pre-linguistic visual processing. Consequently, the $\mathrm{A} \times \mathrm{C}$ activation map represents the activation during the silent reading of words and the $\mathrm{B} \times \mathrm{C}$ map represents activation during the silent reading of nonwords.

Each activation map was composed of 15 
slices (Figure 1). To identify the anatomical localization of the activated areas, each slice was analyzed visually, determining which areas were activated using as reference the Atlas of Duvernoy (25). The activated areas were identified independently using an Atlas by two examiners (MLHS and PC). The points of disagreement between the two analyses were discussed by the examiners, who then reached a consensus. Based on these data, two tables were elaborated, one for each comparison ( $\mathrm{x}$ x $\mathrm{C}$ and $\mathrm{B} \times \mathrm{C}$ ) for the areas activated in each subject. The number of activated voxels was recorded in all active clusters resulting from the $\mathrm{A} \times \mathrm{C}$ and $\mathrm{B} \times \mathrm{C}$ comparisons.

Initially we determined the frequency of activation in different brain areas for each individual in each specific condition. This procedure provided an overview of all possible areas detected in the subjects. Since not all areas were present in all subjects, the frequency of activation detected in each area provides a measure of the commonalities in the neural correlates of each task found in the participants. Areas displaying activation

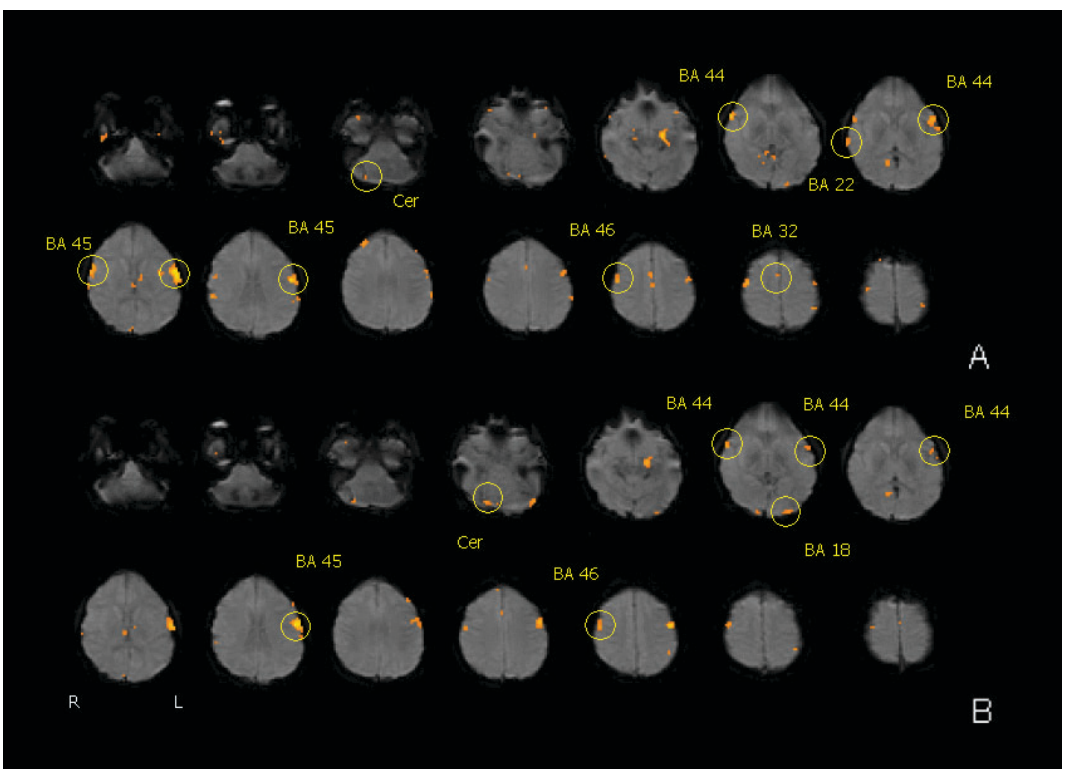

Figure 1. Brodmann areas (BA) are shown in circles for two different analysis from the same subject: $A$, areas detected when comparing real words (lexical processing) versus illegitimate graphic stimuli; $B$, areas detected when comparing nonwords (phonological processing) versus illegitimate graphic stimuli. Cer = cerebellum. in more than $50 \%$ of the individuals were judged to represent relevant activations. This approach has been used in past publications by others, and we believe it represents a compromise between subject variability and group-shared activation foci $(26,27)$.

\section{Results}

The comparison of the number of activated voxels in all individuals in the conditions $\mathrm{A} \times \mathrm{C}$ (words $\mathrm{x}$ illegitimate graphic stimuli) and B x C (nonwords $\mathrm{x}$ illegitimate graphic stimuli) showed a larger number of voxels activated in the first condition, indicating a greater activation in word than in nonword reading. Furthermore, in both conditions the left cerebral hemisphere was more activated than the right hemisphere (Table 2).

Table 3 shows the areas that were activated in more than $50 \%$ of the individuals in the conditions $\mathrm{A} \times \mathrm{C}$ and $\mathrm{B} \times \mathrm{C}$ in the right and left hemispheres. A larger number of activated common areas can be observed in the silent reading of words than of nonwords in both hemispheres. In the first condition, i.e., word silent reading, a mirror correspondence of activations was observed when comparing the left and right hemispheres. The following areas were activated: superior, middle and inferior frontal gyri and the superior temporal gyrus in both cerebral hemispheres. The cerebellum was activated in the right hemisphere and the precentral gyrus in the left. In the condition $\mathrm{B} \times \mathrm{C}$, the right cerebellum and the left superior temporal gyrus were activated in more than $50 \%$ of the individuals.

\section{Discussion}

\section{Differences in the cerebral activation} patterns of lexical and phonological reading

The activation patterns detected in subjects during word and nonword reading were different. In the word reading task, we iden- 
tified ten different activated areas in more than $50 \%$ of the individuals, five of them in the left hemisphere and five in the right. In the reading of nonwords, only two activated areas were found in more than $50 \%$ of the individuals. During word reading, a similar pattern of activation was observed in the left and right hemispheres. In other words, of the five areas activated in each hemisphere, four were activated bilaterally: superior, middle and inferior frontal gyri, and superior temporal gyrus. In addition to these areas, the right cerebellum and the left pre-central gyrus were also activated. In the reading of nonwords, only the right cerebellum and the left superior temporal gyrus were activated. The mirror pattern of activation found in word reading was not observed in nonword reading. The areas activated during the reading of nonwords, the right cerebellum and the left superior temporal gyrus, were also activated during the reading of words.

Petersen et al. (11) and Pugh et al. (20) have also reported different patterns of cerebral activation when comparing the mechanisms involved in word and nonword reading. Petersen et al. (11), in a study with PET, observed that silent reading of words activated the left frontal cortex. The medial extrastriate visual cortex in the left hemisphere was activated during the silent reading of both words and nonwords. In the study of Pugh et al. (20), using fMRI, 38 individuals completed a judgment task of phonological processing (nonword rhyme judgment) and lexical-semantic processing (semantic category judgment). The researchers observed activation during phonological processing in the inferior frontal gyrus and in the temporal lobe (with lateralization of the activation of the inferior frontal gyrus to the left in men, and bilateral activation in women) and activation of the superior and middle temporal gyri in both hemispheres during the lexical-semantic processing. In contrast, in a PET study on 14 right-handed men, Rumsey et al. (14) did not find differ- ences in activation during tasks of lexical and phonological reading. The reading of irregular words as well as of nonwords activated the following areas: left lingual and left fusiform gyri, cerebellum, superior temporal gyrus bilaterally, left thalamus, periRolandic cortex (pre- and post-central gyri) bilaterally, anterior/mid cingulate gyrus on the left, and left insula. Price et al. (13), in another study with PET and reading of real words, found activation during silent reading in the following areas: medial extrastriate cortex on the left, middle temporal cortex on the left, middle frontal cortex on the left, and middle temporal cortex and inferior frontal cortex on the right.

The comparison of our data with those reported by other researchers should be made with caution. One of the reasons for this is related to the use of different tasks to probe

Table 2. Number of voxels activated in the word and nonword silent reading.

\begin{tabular}{lcc}
\hline & $\begin{array}{c}\text { Condition A x C } \\
\text { Words }\end{array}$ & $\begin{array}{c}\text { Condition B x C } \\
\text { Nonwords }\end{array}$ \\
\hline Right cerebral hemisphere & $\begin{array}{l}794 \text { voxels } \\
874 \text { voxels }\end{array}$ & $\begin{array}{l}446 \text { voxels } \\
526 \text { voxels }\end{array}$ \\
\hline
\end{tabular}

Condition $\mathrm{A}=$ list 1 ; condition $\mathrm{B}=$ list 2; condition $\mathrm{C}=$ list 3 . See Table 1 for explanation of lists.

Table 3. Areas activated in more than $50 \%$ of the individuals during the silent word and nonword reading in the right and left hemispheres.

\begin{tabular}{lcc}
\hline & $\begin{array}{c}\text { Condition A x C } \\
\text { Words }\end{array}$ & $\begin{array}{c}\text { Condition B x C } \\
\text { Nonwords }\end{array}$ \\
\hline Right hemisphere & $\begin{array}{c}\text { Superior frontal gyrus (10) } \\
\text { Medium frontal gyrus (10) } \\
\text { Cerebellum (9) } \\
\text { Inferior frontal gyrus (8) } \\
\text { Superior temporal gyrus (7) }\end{array}$ & \\
& Superior frontal gyrus (8) & Superior temporal gyrus (7) \\
& Medium frontal gyrus (7) \\
Inferior frontal gyrus (7) & \\
Superior temporal gyrus (7) \\
Precentral gyrus (7)
\end{tabular}

The numbers in parentheses refer to the number of subjects of the 13 in the study who presented activation in the area. Condition $\mathrm{A}=$ list 1 ; condition $\mathrm{B}=$ list 2 ; condition $\mathrm{C}=$ list 3. See Table 1 for explanation of lists. 
the same function. In the investigations mentioned above, the activation during reading was confirmed through similar but not identical tasks, i.e., silent reading, reading aloud, lexical decision, and judgment. Probably, the execution of these tasks involves similar and different mechanisms. In this way, the cerebral areas activated during the performance of these tasks should be different. Even the sensitivity of the paradigm can influence the data. Price et al. (13) varied the duration of stimulus presentation (150 and $981 \mathrm{~ms}$ ) in tasks of silent reading, reading aloud and lexical decisions and showed that the time of exhibition, significantly influenced the correlation between blood flow and word reading. The divergence of the results obtained in the different studies is an important issue to be considered in studies that involve functional neuroimaging and mental functions and should be addressed by additional studies in the field.

\section{Right cerebral hemisphere and word reading}

An interesting aspect of the results obtained in the present study is the mirror correspondence of the areas that were activated. This pattern was only observed for the silent reading by the lexical route, that is, for the processing of irregular and foreign words. From these findings the following question could be raised: does the right cerebral hemisphere play a role in lexical reading?

In reality this issue has already been discussed in the literature for some years (2830). Some researchers defend the "right hemisphere hypothesis", which proposes that the right cerebral hemisphere plays a role in the orthographic and lexical processes of reading, permitting the processing of high imaginability and high frequency written words. This hypothesis is supported by case studies of left hemispherectomy, split-brain cases and patients with deep dyslexia and pure alexia.

Patterson et al. (31) described the case of patient NI, who, after a normal childhood, developed refractory epilepsy, needing the surgical removal of the left cerebral hemisphere. After the hemispherectomy, NI presented reading residual abilities characteristic of deep dyslexia. She was capable of reading some concrete and high frequency words, making some semantic mistakes. Zaidel and Peters (32) described two splitbrain patients who were able to associate with objects the written word presented to the right hemisphere. Coslett and Saffran (33) reported four patients with pure alexia whose performance in tasks of lexical decision and semantic categorization was consistent with the right hemisphere hypothesis. Some investigators relate the residual reading abilities of patients with deep dyslexia to the orthographic and semantic aspects of reading by the right hemisphere. In this condition, the patients present an extensive lesion of the left hemisphere and are able to read some frequent words through lexical processing, which would be an indication that in these patients with deep dyslexia reading is being accomplished by the right hemisphere.

Coslett and Monsul (29) conducted a study using transcranial magnetic stimulation (TMS) in a case of pure alexia (JG) to test the "right hemisphere hypothesis". The authors compared the reading performance of JG in three different situations: without TMS, with TMS on the right parietal lobe and with TMS in the same area on the left. Having as a base the performance in reading without TMS, the authors compared the results of the influence of TMS on the right and the left parietal lobe and verified that the reading performance of JG was impaired only when the right cerebral hemisphere was stimulated, supporting the idea that the residual reading of that patient was due to a linguistic ability of the non-dominant hemisphere.

The results obtained in the present study regarding the mirror pattern of activation in the left and right cerebral hemispheres dur- 
ing real word reading support this idea that the right cerebral hemisphere has a role in lexical reading.

\section{The role of the cerebellum}

In this study, the right cerebellar hemisphere was activated in both the word and nonword reading tasks in more than half the subjects. These data suggest the participation of the cerebellum in reading, corroborating the findings from the study of Fulbright et al. (34). The contribution of the cerebellum to cognitive processing was discussed by these researchers, who studied the cerebellar activation by fMRI during reading and concluded that the cerebellum is engaged not only in the ability of phonologic assembly but also in semantic processing.

\section{Limitations and perspectives}

As in other studies that use fMRI, one of the limitations of the present study was related to the small number of subjects evaluated. We tried to minimize this problem by selecting right-handed female volunteers with a high educational level to obtain a more homogeneous sample.

Another limitation refers to individual differences in the performance of cognitive tasks. The acquisition of reading, differently from the acquisition of the oral language, is not an innate ability of humans. Since reading is a culturally biased function it might suffer a larger inter-individual variability than the other innate and primary cognitive abilities. In the present study, we chose the silent reading task to determine the pattern of cerebral activation because of our interest in examining the complete process of reading from the input of visual information to phonological codification, without the requirement of speech articulation that could provoke the appearance of artifacts in the fMRI images. Some studies of reading use other tasks, such as lexical decision and semantic categorization of written words, that test some isolated mechanisms involved in reading. Leff et al. (35) consider silent reading to be a natural function, different from tasks of lexical decision or of choice, that imply the activation of other associated mental processes difficult to control for each individual. If, on the one hand, it seems to have advantages over the choice of the silent reading task, it also has disadvantages. When using the silent reading task during image acquisition by fMRI we cannot be certain of the volunteer's collaboration at the moment of scanning. In the present study, to confirm that the silent reading task was actually performed, soon after the experiment, individuals had to mention some stimuli that had been presented.

Some studies have investigated cerebral activation using a limited number of slices, without a global vision of the brain. In our study, the 15 slices covered the whole brain. This type of technical approach became available recently. It facilitates not only the observation of the activation of areas of interest, but also the inspection of all the areas of the brain.

In spite of the limitations and controversies generated from the cerebral activation maps related to the cognitive abilities, studies with the use of fMRI contribute and will continue to contribute to the understanding of the cognitive and neural mechanisms related to the mental functions. fMRI, together with other experimental approaches using advanced techniques like PET and TMS, as well as lesion studies, should provide relevant information about reading processes.

According to our hypothesis, the results reported here concerning brain activation demonstrate differences in the activation patterns associated with lexical and phonological reading. However, we believe it is still too early to know if these different patterns correspond to two independent neural networks, one responsible for the lexical processing of reading and the other for the phonological processing. 


\section{Acknowledgments}

We wish to thank Antônio Cesário Monteiro da Cruz Junior for providing the elec- tronic instruments necessary for synchronizing stimulus presentation and data acquisition, and for the network configuration.

\section{References}

1. Hécaen H \& Albert ML (1977). Human Neuropsychology. WileyInterscience, New York.

2. Black SE \& Behrmann M (1994). Localization in alexia. In: Kertesz A (Editor), Localization and Neuroimaging in Neuropsychology. Academic Press, San Diego, CA, USA, 331-376.

3. Lecours AR, Delgado AP \& Pimenta MAM (1993). Distúrbios adquiridos da leitura e da escrita. In: Mansur LL \& Rodrigues N (Editors), Temas em Neurolingüística. Tec Art, São Paulo, SP, Brazil, 45-62.

4. McCarthy RA \& Warrington EK (1990). Cognitive Neuropsychology: A Clinical Introduction. Academic Press, San Diego, CA, USA.

5. Lesser R \& Milroy L (1993). Linguistics and Aphasia: Psycholinguistic and Pragmatic Aspects of Intervention. Longman, New York, 5280.

6. Ellis AW \& Young AW (1988). Human Cognitive Neuropsychology. Lawrence Erlbaum, London, UK.

7. Price CJ (2000). Functional imaging studies of aphasia. In: Mazziotta JC, Toga AW \& Frackowiak RS (Editors), Brain Mapping: the Disorders. Academic Press, San Diego, CA, USA, 181-200.

8. Basso A (2000). The aphasias: fall and renaissance of the neurological model? Brain and Language, 71: 15-17.

9. Marshall JC \& Newcombe M (1973). Patterns of paralexia: a psycholinguistic approach. Journal of Psycholinguistic Research, 2: 175-200.

10. Caramelli P, Parente MAMP, Hosogi ML et al. (1994). Unexpected reading dissociation in a Brazilian "nisei" with crossed aphasia. Behavioural Neurology, 7: 165-170.

11. Petersen SE, Fox PT, Snyder AZ et al. (1990). Activation of extrastriate and frontal cortical areas by visual words and word like stimuli. Science, 249: 1041-1044.

12. Small SL, Flores DK \& Noll DC (1998). Different neural circuits subserve reading before and after therapy for acquired dyslexia. Brain and Language, 62: 298-308.

13. Price CJ, Wise RJ, Watson DG et al. (1994). Brain activity during reading. The effects of exposure duration and task. Brain, 117: 1255-1269.

14. Rumsey JM, Horwitz B, Donohue BC et al. (1997). Phonological and ortographic components of word recognition: a PET-rCBF study. Brain, 120: 739-759.

15. Henson RNA, Burgess N \& Frith CD (2000). Recoding, storage, rehearsal and grouping in verbal short-term memory: an fMRI study. Neuropsychologia, 38: 426-440.

16. Price CJ, Gorno-Tempini ML, Graham KS et al. (2003). Normal and pathological reading: converging data from lesion and imaging studies. Neuroimage, 20: S30-S41.

17. Coltheart M, Patterson KE \& Marshall JC (Editors) (1980). Deep Dyslexia. Routledge, Londres, UK.
18. Coltheart M, Masterson J, Byng S et al. (1983). Surface dyslexia. Quarterly Journal of Experimental Psychology, 35A: 469-495.

19. Lecours AR \& Parente MAMP (1997). Dislexia: Implicações do Sistema de Escrita do Português. Artes Médicas, Porto Alegre, RS, Brazil.

20. Pugh KR, Shaywitz BA, Shaywitz SE et al. (1996). Cerebral organization of component process in reading. Brain, 119: 1221-1238.

21. Oldfield RC (1971). The assessment and analysis of handedness: the Edinburgh Inventory. Neuropsychologia, 9: 97-113.

22. Bullmore E, Brammer MJ, Rabe-Hesketh S et al. (1999). Methods for diagnosis and treatment of stimulus-correlated motion in generic brain activation studies using fMRI. Human Brain Mapping, 7: 38-48.

23. Bullmore E, Long $\mathrm{C}$, Suckling $\mathrm{J}$ et al. (2001). Colored noise and computational inference in neurophysiological (fMRI) time series analysis: resampling methods in time and wavelet domains. Human Brain Mapping, 12: 61-78.

24. Bullmore E, Fadili J, Breakspear M et al. (2003). Wavelets and statistical analysis of functional magnetic resonance images of the human brain. Statistical Methods in Medical Research, 12: 375-399.

25. Duvernoy HM (1991). The Human Brain-Surface, Three-Dimensional Sectional Anatomy and MRI. Springer-Verlag, Vienna, Austria.

26. Uchida I, Kikyo H, Nakajima K et al. (1999). Activation of lateral extra-striate areas during orthographic processing of Japanese characters studied with fMRI. Neuroimage, 9: 208-215.

27. Wood AG, Harvey AS, Wellard RM et al. (2004). Language cortex activation in normal children. Neurology, 63: 1035-1044.

28. Coltheart M (2000). Deep dyslexia is right-hemisphere reading. Brain and Language, 71: 299-309.

29. Coslett HB \& Monsul N (1994). Reading with the right hemisphere: evidence from transcranial magnetic stimulation. Brain and Language, 46: 198-211.

30. Ellis AW (1995). Leitura, Escrita e Dislexia: Uma Análise Cognitiva. Artes Médicas, Porto Alegre, RS, Brazil.

31. Patterson K, Vargha-Khadem F \& Polkey C (1988). Reading with one hemisphere. Brain, 112: 39-63.

32. Zaidel E \& Peters AM (1981). Phonological encoding and ideographic reading by the disconnected right hemisphere: two case studies. Brain and Language, 14: 205-234.

33. Coslett HB \& Saffran EM (1989). Evidence for preserved reading in "pure alexia". Brain, 113: 327-329.

34. Fulbright RK, Jenner AR, Mencl WE et al. (1999). The cerebellum's role in reading: a functional MR imaging study. American Journal of Neuroradiology, 20: 1925-1930.

35. Leff AP, Crewes H, Plant GT et al. (2001). The functional anatomy of single-word reading in patients with hemianopic and pure alexia. Brain, 124: 510-521. 